\title{
Synthesis and characterization of novel cardanol based fulleropyrrolidines
}

\author{
Orazio A. Attanasi, ${ }^{a}$ Giuseppe Mele, ${ }^{* b}$ Paolino Filippone, ${ }^{a}$ Selma E. Mazzetto, ${ }^{c}$ and \\ Giuseppe Vasapollo ${ }^{b}$ \\ ${ }^{a}$ Centro di Studio delle Sostanze Naturali, Università degli Studi di Urbino “Carlo Bo”, Via \\ Sasso 75, 61029 Urbino, Italy \\ ${ }^{b}$ Dipartimento di Ingegneria dell'Innovazione, Università del Salento, Via Arnesano, 73100 \\ Lecce, Italy \\ ${ }^{c}$ Departamento de Química Orgânica e Inorgânica - Universidade Federal do Ceará-UFC, \\ Caixa Postal 12.200, 60455-760 Fortaleza ,CE - Brazil \\ E-mail: giuseppe.mele@unile.it
}

This paper is dedicated to Professor Nicolò Vivona on the occasion of his 70th anniversary

\begin{abstract}
Cardanol oil, a renewable raw material well known by product of the cashew industry, has been used as the starting material for the synthesis of novel fulleropyrrolidines cardanol based. In this work, cardanol has been used as building block for the preparation of target cardanol based precursors obtained by the way of the convenient transformation of the functional groups (aromatic ring, -OH group or the double bonds of the side chain) of the cardanolic structure. Pure $3-n$-pentadecylphenol and its derivatives having homogeneous chemical composition, used as the precursor of any fulleropyrrolidines, have been prepared by hydrogenation of the un-saturated side chain and subsequent alkylation of the aromatic ring of cardanol. The reactivity of olefinic double bond present in the side-chain which can undergo easy transformation i.e. oxirane formation as well as metathesis reactions affording various interesting fulleropyrrolidines is also described.
\end{abstract}

Keywords: Cardanol, fullerene, fulleropyrrolidines, metathesis, 3-n-pentadecylphenol

\section{Introduction}

Recycling of renewable organic wastes to produce new fine chemicals is not a new concept. In fact, "from waste to value" is a well know phrase, following the basic idea to synthesize new molecules using secondary materials and by-products from industry. 
Cardanol is an industrial grade yellow oil obtained by vacuum distillation of "Cashew Nut Shell Liquid" (CNSL), the international name of the alkyl phenolic oil contained in the spongy mesocarp of the cashew nut shell (Anacardium occidentale L.). ${ }^{1}$ CNSL derives from the most diffused roasted mechanical processes of the cashew industry, represents nearly $25 \%$ of the total nut weight and its production in the worldwide (Africa, Asia, and South America being the main producer areas) is estimated to be about 300,000 tons per year. As shown as Figure 1, CNSL is a mixture of anacardic acid, cardanol, and traces of cardol and 2-methylcardol. The alkyl side chain $(\mathrm{R})$ of each of them may be saturated, monolefinic $(8)$, diolefinic $(8,11)$ and triolefinic $(8$, $11,14) .{ }^{1}$ Due to the decarboxylation of anacardic acid during the distillation process, cardanol results to be the main component of distilled CNSL.

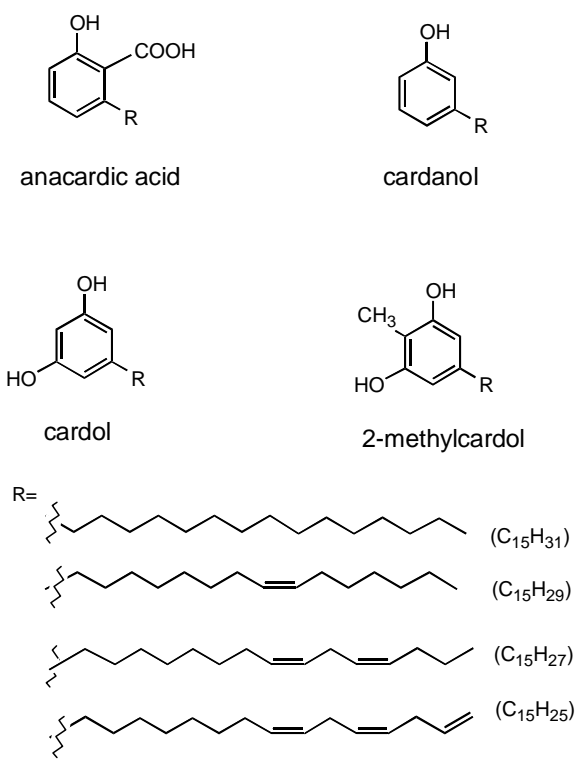

Figure 1. Components of Cashew Nut Shell Liquid.

Cardanol itself is a mixture of compounds having a variable number of unsaturations in the fifteen carbon atom chains with mainly one double bond per molecule. ${ }^{1}$ In the last years, cardanol has become an important building-block used for the construction of an increasing number of new organic molecules.

In the last decade functionalisation of $\mathrm{C}_{60}$ has attracted the interest of scientists for the possibility to obtain new fullerene based hybrid functional materials for technological purposes. ${ }^{2-10}$

On the other hand, fullerenes, which can be considered the older nanomaterials relative of carbon nanotubes, continue to stimulate advances in applied and fundamental science. As well known, fullerenes are excellent electron acceptors and can be chemically modified to improve solubility in organic solvents by the presence appropriate bulking substituents and functional groups. Recently, particular attention has been paid for the preparation of hybrid systems between natural compounds -such as steroids, carbohydrates ${ }^{11}$ and flavonols, ${ }^{12,13}$ amino acid 
derivatives, ${ }^{14}$ and fullerene due to their potential application in medicinal chemistry, pharmaceuticals and so on. A few years ago, it has been reported that, when in the fullerene derivatives is present a long alkyl chain the fulleropyrrolidine derivatives showed considerable solubility in the organic solvents. ${ }^{15,16}$ Owing to the difficulty of synthesizing phenols with a long unsaturated chain -like the fifteen carbon atom chain in the meta position- cardanol represents a peculiar, simple and easily available and precious precursor used as starting material for the synthesis of various derivatives. Several functionalizations of cardanol moiety have been obtained by reactions of the aromatic ring, hydroxy group (i.e. alkylation, nitration etc.) as well as the unsaturations on the chain in the meta position. ${ }^{17}$ These reasons prompted us to synthesize and characterize novel cardanol based fulleropyrrolidines starting from cardanol precursors.

\section{Results and Discussion}

Cardanol oil, obtained by vacuum distillation of CNSL, was redistilled twice using a Vigreux column. Three different fractions were separately collected and the second one was used as starting material for the preparation of cardanol precursors for the fulleropyrrolidine derivatives, reported in this work. GC-MS and NMR analyses were carried out in order to determine the composition of the fractions. They confirmed that the mono unsaturated compound, 3-npentadeca-8-enylphenol, 1a, was the main component of the second fraction; with small amounts $(<1 \%)$ of saturated, di- and tri-olefinic components (respectively, 3- $n$-pentadecylphenol, 3- $n$ pentadeca-8,11-dienylphenol, and 3-n-pentadeca-8,11,14-trienylphenol).

Hydrogenated cardanol 1b (3-n-pentadecylphenol) can be easily obtained as pure compound by hydrogenation of the double bond(s), present in the side-chain, of technical grade distilled cardanol and subsequent distillation and/or crystallization. In this way, several functionalizations of the aromatic ring or hydroxy group (i.e. alkylation) can be performed without undesirable side-reactions ascribable to olefinic moiety. Compound $\mathbf{1 b}$, successively underwent to alkylation reactions, gave the compounds $\mathbf{1 c}, \mathbf{1 d}$ (Scheme 1). ${ }^{18}$

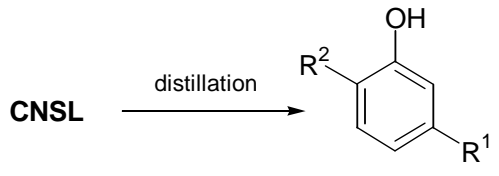

$1 \mathbf{a}$

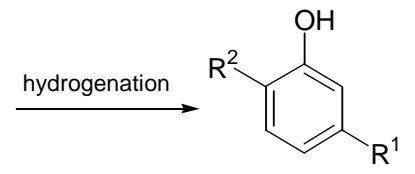

1b

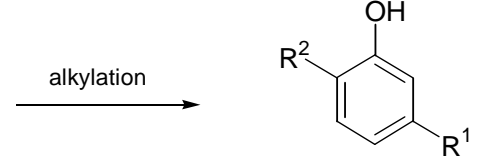

1c, 1d

1a: $\mathrm{R}^{1}=\mathrm{C}_{15} \mathrm{H}_{29} \quad \mathrm{R}^{2}=\mathrm{H} ; \mathbf{1 b}: \mathrm{R}^{1}=\mathrm{C}_{15} \mathrm{H}_{31} \quad \mathrm{R}^{2}=\mathrm{H}, \mathbf{1 c}: \mathrm{R}^{1}=\mathrm{C}_{15} \mathrm{H}_{31} \quad \mathrm{R}^{2}=t$-butyl; 1d: $\mathrm{R}^{1}=\mathrm{C}_{15} \mathrm{H}_{31} \quad \mathrm{R}^{2}=t$-amyl

Scheme 1. Production processes of the cardanol-based precursors (1a-1d) from CNSL.

Compounds 1a-1d were used as starting materials for the preparation of the aldehyde precursors 5a-5e which effectively reacted with $\mathrm{C}_{60}$ to obtain the new fulleropyrrolidines reported in this paper. So that, cardanol derivatives 3a-3d were prepared in almost quantitative 
yield reacting 1a-1d with 1,2-dibromoethane (2), in the presence of potassium hydroxide at $70{ }^{\circ} \mathrm{C}$. Successively, 3a-3d were converted in good yields (40-60\%) into their respective aldheydic derivatives 5a-5d by reaction, under reflux, with 4-hydroxybenzaldehyde (4) in acetone and in the presence of potassium carbonate (Scheme 2).

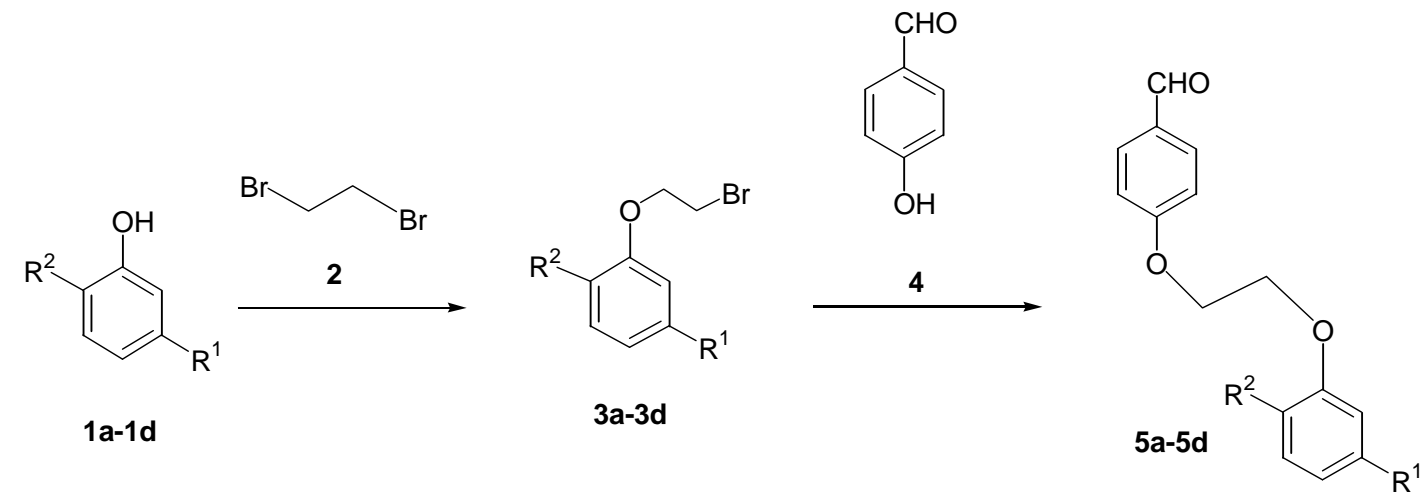

1a, 3a, 5a: $\mathrm{R}^{1}=\mathrm{C}_{15} \mathrm{H}_{29} \quad \mathrm{R}^{2}=\mathrm{H}$; 1b, 3b, 5b: $\mathrm{R}^{1}=\mathrm{C}_{15} \mathrm{H}_{31} \quad \mathrm{R}^{2}=\mathrm{H}$, 1c, 3c, 5c: $\mathrm{R}^{1}=\mathrm{C}_{15} \mathrm{H}_{31} \quad \mathrm{R}^{2}=$ t-butyl; 1d, 3c, 5c: $\mathrm{R}^{1}=\mathrm{C}_{15} \mathrm{H}_{31} \quad \mathrm{R}^{2}=t$-amyl

Scheme 2. Synthesis of the aldehyde derivatives 5a-5d from the starting materials cardabolbased 1a-1d.

The olefinic double bond(s) present in the side-chain of cardanol (Scheme 2) seems don't have any influence in the reaction with 1,2-dibromoethane, under the above-mentioned conditions. Also, the-olefinic double bond present in the 8 position of the-chain in 1a, difficult to synthesize by other procedures, kept the natural position in the course of the various transformations. The mono-olefinic derivative 3a was isolated in this way as the main component in form of orange oil and then converted into the corresponding aldheydic derivative 5a in $40 \%$ isolated yield. The presence of a double bond on the side chain of cardanolic molecule permitted us also to prepare the corresponding oxirane derivative. In fact, the aldheydic derivative 5a was reacted with $m$-chloroperbenzoic acid (MCPBA) in dichloromethane at $0{ }^{\circ} \mathrm{C}$ producing the mono epoxide 5 e in $70 \%$ yields as sticky white solid (Scheme 3 , path a).

Also, due to the presence of a double bond on the side chain, cardanol, and some of its derivatives, have been recently used as starting materials to perform olefin cross metathesis reactions by using different generation of Grubbs' catalysts. ${ }^{19}$ In particular, the Ru-carbene catalyst named C627, which structure is reported in Figure 2, resulted the most efficient to perform homo-cross-metathesis (HCM) of cardanol based olefins. 


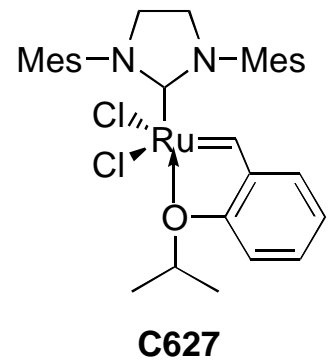

Figure 2. Structure of $\mathbf{C 6 2 7}$ used as catalyst for homo-cross-metathesis reactions.

The homo-cross metathesis reaction of cardanol derivative 5a (Scheme 3, path b), carried out according with previously reported reaction conditions, permitted us to obtain the biscardanol derivative $5 \mathbf{f}$ in good yields. ${ }^{19}$

All the compounds 3a-3d and 5a-5e were characterized by FT-IR, MS, ${ }^{1} \mathrm{H}$ and ${ }^{13} \mathrm{C}$ NMR analysis. The spectra were in perfect agreement with the proposed structure. In particular, ${ }^{1} \mathrm{H}$ NMR spectra of 3a and $\mathbf{5 a}$ in $\mathrm{CDCl}_{3}$ showed the signals corresponding to the double bonds of cardanol side chain (in the range of $\delta=5.39-5.37 \mathrm{ppm}$ ) besides the other signals typical of the hydrogenated derivatives $\mathbf{3 b}-\mathbf{3 d}$ and $\mathbf{5 b}-\mathbf{5 d}$. ${ }^{1} \mathrm{H}$ NMR spectrum of compound $5 \mathbf{e}$ showed the signals corresponding to protons of the epoxy moiety $(\delta=2.99-3.01 \mathrm{ppm})$ and to the protons of the $\mathrm{CH}_{2}-7$ and $\mathrm{CH}_{2}-10$ ( $\delta=2.84$ and 2.96 ppm respectively); ${ }^{1} \mathrm{H}$ NMR spectrum showed also the disappearance of the signals corresponding to the double bonds. ${ }^{13} \mathrm{C}$ NMR spectrum of $5 \mathbf{e}$ showed the signal typical of the oxiranic carbons at $\delta=58 \mathrm{ppm}$. The compounds 5a-5e were used as useful tools for the synthesis of novel fulleropyrrolidine derivatives.

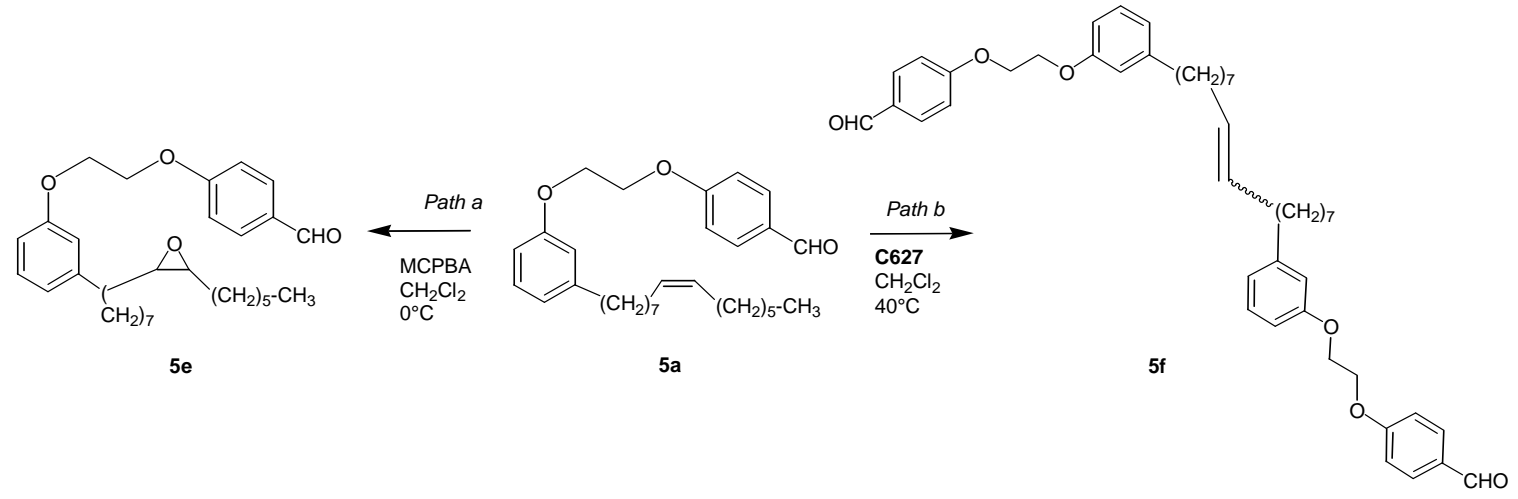

Scheme 3. Oxirane formation (path $a$ ) and metathesis (path $b$ ) reactions involving the double bond on the side chain of the mono unsaturated cardanol molecule.

Usually, the synthesis of the fulleropyrrolidines has been carried out via condensation of aminoacids (e.g. of $N$-methylglycine, $N$-phenylglycine) with aldehyde compounds and $\mathrm{C}_{60}$ through a typical 1,3-dipolar addition of azomethine ylides generated in situ. ${ }^{[20]}$ So, as shown in Scheme 4 , the synthesis of the fulleropyrrolidines $7 \mathbf{a}-7 \mathbf{i}$ were performed through a cycloaddition 
to $\mathrm{C}_{60}$ of the azomethyne ylide resulting from the decarboxylation of $N$-methylglycine $6 \mathbf{a}$ or $N$ phenylglycine $\mathbf{6 b}$ in the presence of the cardanol aldheydidic precursors 5a-5f. In particular, 5a, $\mathrm{C}_{60}$ and $\mathrm{N}$-methylglycine were allowed to react in refluxing toluene for $24 \mathrm{~h}$. After cooling, the resulting solution was evaporated to dryness and the residue purified by column chromatography (silica, toluene) yielding 7a in 33\%. All the products were however purified by column chromatography on silica and characterized by FT-IR, LC/MS, ${ }^{1} \mathrm{H}$ and ${ }^{13} \mathrm{C}$ NMR analysis. The ${ }^{1} \mathrm{H}$ NMR spectra of the compounds $7 \mathbf{a}-7 \mathbf{i}$ were in agreement with the proposed structures and showed typical signals of the pyrrolidine $\left(\mathrm{C}_{60}\right)$-fullerene system: a singlet for $\mathrm{H}-2$ at $\delta=4.92$ ppm and the $\mathrm{AB}$ system for $\mathrm{CH}_{2}-5$, two doublets centered respectively at $\delta=4.27$ ppm and $\delta=$ $5.00 \mathrm{ppm}$ with a geminal coupling $J_{\mathrm{AB}}=9.4 \mathrm{~Hz}$. In particular, ${ }^{1} \mathrm{H}$ NMR spectrum of compound $7 \mathrm{~g}$ showed the signals corresponding to the proton of the double bound at $\delta=5.38 \mathrm{ppm}$ while ${ }^{1} \mathrm{H}$ NMR spectrum of compound $\mathbf{7 h}$ showed the signals corresponding to proton of the $\mathrm{CH}_{2}-6$ and $\mathrm{CH}_{2}-9$ of the cardanol side chain at $\delta=2.84-2.96$ ppm respectively.
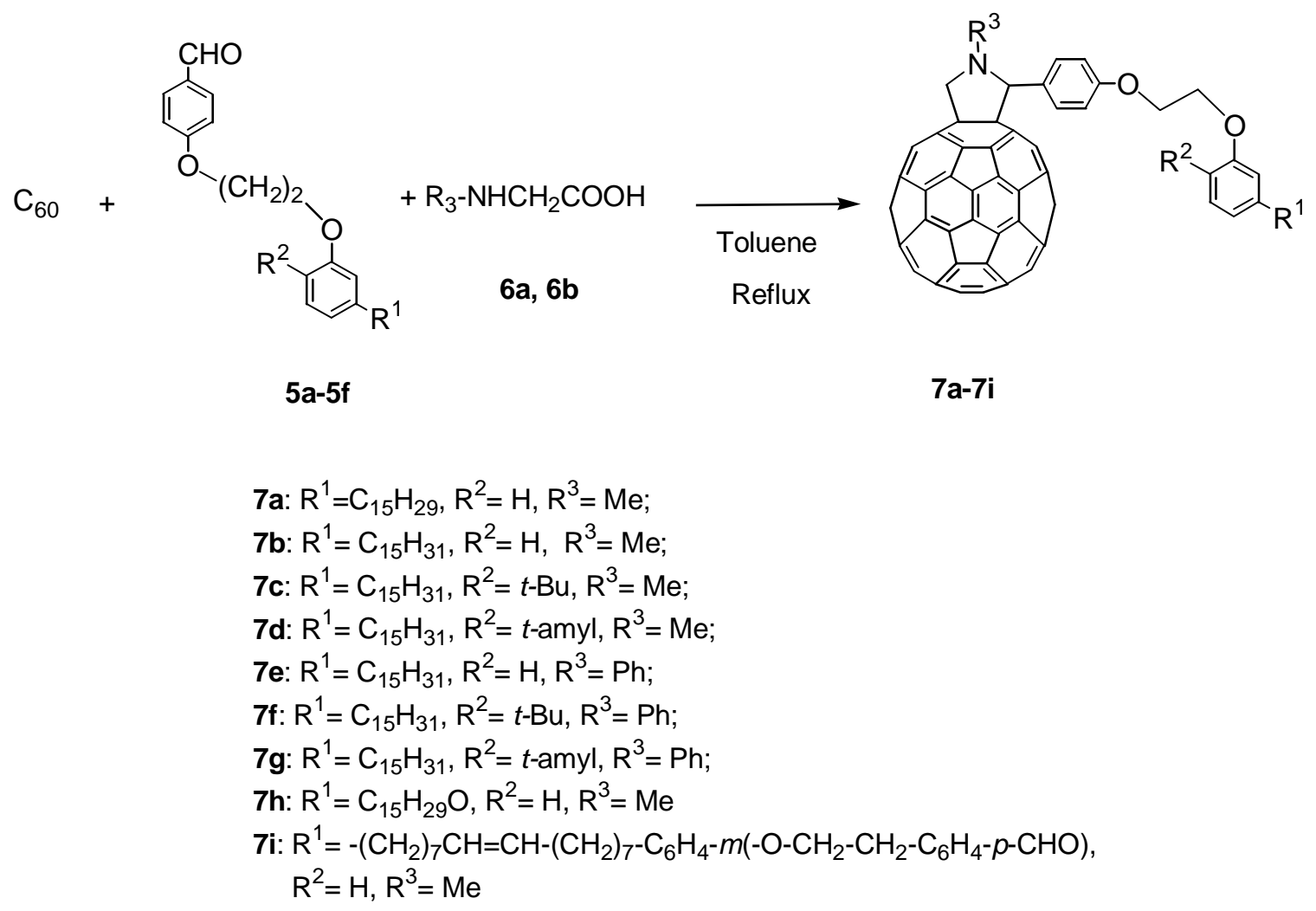

Scheme 4. Synthesis of fulleropyrrolidines 7a-7i cardanol-based. 

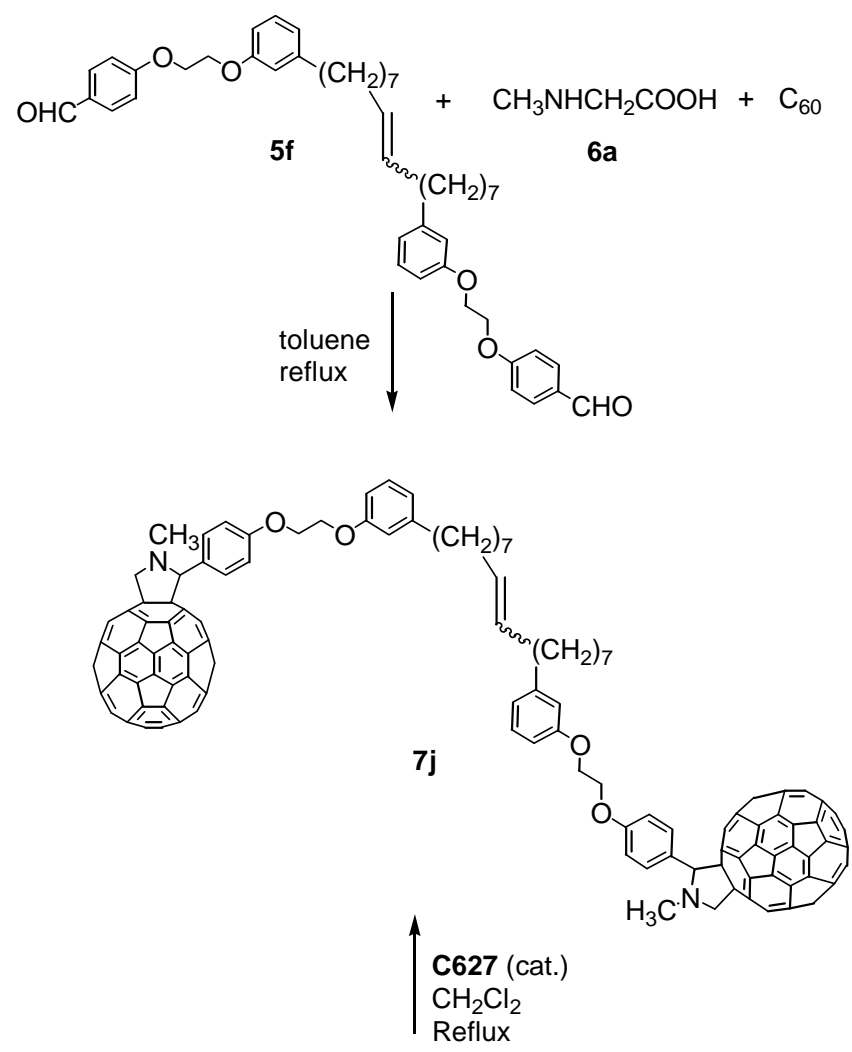

$7 i$

Scheme 5. Synthesis of the bis-fulleropyrrolidine $\mathbf{7 j}$ by different pathways: path a) starting from the metathesis product $\mathbf{5 f}$; path $b$ ) by metathesis reaction of the fulleropyrrolidine $\mathbf{7 i}$.

The signals in the ${ }^{13} \mathrm{C}$ NMR spectra of the compounds $7 \mathbf{a}-7 \mathbf{i}$ are also in agreement with the proposed structures. The number of signals in the range around 136-159 ppm (136-168 in the case of compound $\mathbf{7 h}$ ) shows the lack of symmetry typical of asymmetric fulleropyrrolidine system. ${ }^{13} \mathrm{C}$ NMR spectrum of compound $7 \mathbf{h}$ shows also the signals at $\delta=58$ ppm corresponding to oxiranic carbons. All the analytical and spectral data obtained for $\mathbf{7 a - 7 h}$ were consistent with the compounds. As reported for cardanol-based phthalocyanines and porphyrins, ${ }^{[21,22]}$ cardanolbased fulleropyrrolidines 7a-7i have shown also relatively low melting points and high solubility in the most common organic solvents ascribable certainly to the presence of the long alkyl chains. It is well known that similar fulleropyrrolidines derivatives without adequate substituents are insoluble in organic solvents. Bis-fulleropyrrolidine $7 \mathbf{j}$ was synthesized (Scheme 5) in moderate yields following two different synthetic strategies. In the first one, $\mathbf{7 j}$ was synthesized through a cycloaddition of the bis-azomethyne ylide resulting from the decarboxylation of $N$ methylglycine 6a in the presence of the cardanol based aldehyde precursors $5 \mathbf{f}$ using to $\mathrm{C}_{60}$ in excess; in the second strategy $\mathbf{7} \mathbf{j}$ was instead synthesized through homo-cross metathesis reaction of the fulleropyrrolidine derivative $7 \mathbf{i}$ using Grubbs catalyst $\mathbf{C 6 2 7}$ according with the procedure reported in the experimental section. Unfortunately, the relatively low solubility of $\mathbf{7} \mathbf{j}$, in organic 
solvent (probably due to the presence of two fullerene groups) do not allowed the ${ }^{13} \mathrm{C}-\mathrm{NMR}$ and LC_MS characterization of $\mathbf{7 j}$.

\section{Conclusions}

Novel cardanol-fulleropyrrolidine hybrids have been synthesized starting from cardanol by means of the preparation of ad hoc precursors of cardanol. Cardanol has a phenolic structure substituted in meta position with a long alkyl chain not easily obtainable by synthetic routes. The presence of a double bond in the side chain of cardanol derivatives permitted further transformation (e.g. the introduction of an oxirane functional group or metathesis reactions). Pure 3-n-pentadecylphenol, $\mathbf{1 b}$, and its derivatives of homogeneous chemical composition have been easily prepared by simple hydrogenation of cardanol and subsequent alkylation and transformed without undesirable side-reactions- into the novel precursors able to give fulleropyrrolidines. It is important to remark that the presence of long alkyl chains in fulleropyrrolidine systems improves significantly their solubility in organic solvents.

\section{Experimental Section}

General Procedures. All the starting materials were purchased from Aldrich Chemical Co and used as received. Stabilcardo, a distilled technical grade cardanol provided by Oltremare S.P.A. (Bologna, Italy), has been used as a base compound in this study. Silica gel (Merck) was used in the chromatographic separations. Solvents were dried and distilled under an atmosphere of dry nitrogen. Melting points were taken on an electro thermal apparatus. FT-IR spectra were recorded on a JASCO FT-IR 660 Plus spectrometer. ${ }^{1} \mathrm{H}$ and ${ }^{13} \mathrm{C}$ NMR spectra were recorded on a Bruker Avance 400 at room temperature and chemical shifts are reported relative to tetramethylsilane. Mass spectrometry analyses were carried out by using a LC mass spectrometer 1100 Series (Agilent) equipped with an Electrospray (ESI) interface. Elemental analyses (H, C and N) were performed by Euro Vector (HCNS) instrument.

\section{General procedure for the synthesis of compounds 3a-3d}

Compounds 1a-1d $(13.25 \mathrm{mmol})$ were heated with stirring at $70^{\circ} \mathrm{C}$ with 1,2 -dibromoethane (2) $(15.1 \mathrm{ml}, 175.10 \mathrm{mmol})$. After complete dissolution of 1a-1d, potassium hydroxide $(1.15 \mathrm{~g}$, $19.83 \mathrm{mmol}$ ) was added to the solution and the mixture was stirred for $6 \mathrm{~h}$. The progress of the reaction was monitored by TLC analysis. The mixture was cooled to room temperature and filtered to remove the colorless solid formed. The filtered solution was purified by chromatography on a silica gel column, eluting with $\mathrm{Et}_{2} \mathrm{O}$ /petroleum ether (3:7) to obtain 3a-d in $70-90 \%$ yields. 
1-(2-Bromo-ethoxy)-3-pentadecen-8-yl-benzene. Orange liquid; Yield: 90\% . FT-IR (neat): 2925, 2854, 1662, 1599, 1588, 1484, 1456, 1386, 1258, 1154, 1063, 947, 913, $873 \mathrm{~cm}^{-1}$. MS (EI, $70 \mathrm{eV}): \mathrm{m} / \mathrm{z}(\%)=410(24)\left[\mathrm{M}^{+}+2\right], 408(23)\left[\mathrm{M}^{+}\right], 255$ (15), 228 (17), 214 (100), 107 (57), 79 (26), 55 (24). ${ }^{1} \mathrm{H}$ NMR $\left(\mathrm{CDCl}_{3}, 400 \mathrm{MHz}\right): \delta=0.94$ (t, $\left.J=6.8 \mathrm{~Hz}, 3 \mathrm{H}\right), 1.26-1.44(\mathrm{~m}, 16 \mathrm{H})$, $1.64(\mathrm{~m}, 2 \mathrm{H}), 2.04-2.07(\mathrm{~m}, 4 \mathrm{H}), 2.62(\mathrm{t}, J=7.7 \mathrm{~Hz}, 2 \mathrm{H}), 3.67(\mathrm{t}, J=7.4 \mathrm{~Hz}, 2 \mathrm{H}), 4.32(\mathrm{t}, J=$ $7.4 \mathrm{~Hz}, 2 \mathrm{H}), 5.38-5.44(\mathrm{~m}, 2 \mathrm{H}), 6.75-6.79(\mathrm{~m}, 2 \mathrm{H}), 6.85(\mathrm{~d}, J=7.5 \mathrm{~Hz}, 1 \mathrm{H}), 7.23(\mathrm{t}, J=7.8$ $\mathrm{Hz}, 1 \mathrm{H}) \mathrm{ppm} .{ }^{13} \mathrm{C} \mathrm{NMR}\left(\mathrm{CDCl}_{3}, 400 \mathrm{MHz}\right): \delta=14.56,23.10,27.63,27.66,29.43,29.72,29.77$, 29.90, 30.05, 30.09, 31.78, 31.96, 32.22, 36.43, 68.17, 112.02, 115.48, 122.06, 129.73, 130.25, 130.39, 145.23, 158.53 ppm. Anal. Calc. for $\mathrm{C}_{23} \mathrm{H}_{37} \mathrm{BrO}$ : C, 67.48; H, 9.05. Found: C, 67.49; H, $9.06 \%$.

1-(2-Bromo-ethoxy)-3-pentadecyl-benzene. Colorless powder. Yield: $88 \%$. mp 43-44 ${ }^{\circ} \mathrm{C}$. FTIR (neat): 2922, 2852, 1603, 1584, 1487, 1447, 1255, 1157, 1024, $874 \mathrm{~cm}^{-1}$. MS (EI, $\left.70 \mathrm{eV}\right): \mathrm{m} / \mathrm{z}$ $(\%)=412(35)\left[\mathrm{M}^{+}+2\right], 410(34)\left[\mathrm{M}^{+}\right], 217(10), 216(95), 215$ (36), 214 (100). ${ }^{1} \mathrm{H}$ NMR $\left(\mathrm{CDCl}_{3}, 400 \mathrm{MHz}\right): \delta=0.85(\mathrm{t}, J=6.8 \mathrm{~Hz}, 3 \mathrm{H}), 1.25-1.27(\mathrm{~m}, 24 \mathrm{H}), 1.57(\mathrm{qn}, J=7.6 \mathrm{~Hz}, 2 \mathrm{H})$, $2.55(\mathrm{t}, J=7.6 \mathrm{~Hz}, 2 \mathrm{H}), 3.60(\mathrm{t}, J=6.3 \mathrm{~Hz}, 2 \mathrm{H}), 4.25$ (t, $J=6.3 \mathrm{~Hz}, 2 \mathrm{H}), 6.67-6.70(\mathrm{~m}, 1 \mathrm{H})$, 6.71-6.73 (m, $1 \mathrm{H}), 6.76-6.80(\mathrm{~m}, 1 \mathrm{H}), 7.16(\mathrm{t}, J=7.8 \mathrm{~Hz}, 1 \mathrm{H}) \mathrm{ppm} .{ }^{13} \mathrm{C} \mathrm{NMR}\left(\mathrm{CDCl}_{3}, 400\right.$ MHz): $\delta=14.58,23.15,29.64,29.77,29.78,29.82,29.96,29.99,30.04,30.11,30.12,30.14$, $30.15,31.82$, 32.38, 36.43, 68.17, 112.02, 115.48, 122.06, 129.69, 145.28, 158.51 ppm. Anal. Calc. for $\mathrm{C}_{23} \mathrm{H}_{39} \mathrm{BrO} \mathrm{C}$ : 67.14; $\mathrm{H}$ : 9.55. Found: C, 67.15; H, $9.57 \%$.

1-(2-Bromo-ethoxy)-2-tert-butyl-5-penthadecyl-benzene. Colourless oil. Yield: 75\%. FT-IR (neat): 2922, 2852, 1611, 1504, 1416, 1250, 1179, 1088, 1022, $817 \mathrm{~cm}^{-1}$. MS (EI, $\left.70 \mathrm{eV}\right): \mathrm{m} / \mathrm{z}$ $(\%)=468(14)\left[\mathrm{M}^{+}+2\right], 466(15)\left[\mathrm{M}^{+}\right], 454(20), 453$ (76), 452 (22), $451(73), 215$ (7), $213(7)$, 161 (10), 147 (24), 133 (16), 121 (11), 109 (13), 107 (20), 105 (12), 91 (13), 71 (15), 57 (100), 55 (29). ${ }^{1} \mathrm{H} \mathrm{NMR}\left(\mathrm{CDCl}_{3}, 400 \mathrm{MHz}\right): \delta=0.91(\mathrm{t}, J=6.8 \mathrm{~Hz}, 3 \mathrm{H}), 1.28-1.40(\mathrm{~m}, 24 \mathrm{H}), 1.42(\mathrm{~s}$, $9 \mathrm{H}), 1.60$ (qn, $J=7.6 \mathrm{~Hz}, 2 \mathrm{H}), 2.58(\mathrm{t}, J=7.6 \mathrm{~Hz}, 2 \mathrm{H}), 3.75(\mathrm{t}, J=6.1 \mathrm{~Hz}, 2 \mathrm{H}), 4.36(\mathrm{t}, J=$ $6.1 \mathrm{~Hz}, 2 \mathrm{H}), 6.66(\mathrm{~d}, J=1.0 \mathrm{~Hz}, 1 \mathrm{H}), 6.77(\mathrm{dd}, J=7.9 \mathrm{~Hz}, J=1 \mathrm{~Hz}, 1 \mathrm{H}), 7.22(\mathrm{~d}, J=7.9 \mathrm{~Hz}$, $1 \mathrm{H}) \mathrm{ppm} .{ }^{13} \mathrm{C} \mathrm{NMR}\left(\mathrm{CDCl}_{3}, 400 \mathrm{MHz}\right): \delta=14.55,23.12,29.64,29.77,29.79,29.84,29.91$, 29.97, 30.04, 30.08, 30.12, 30.42, 31.87, 32.35, 34.97, 36.09, 67.99, 112.42, 121.02, 127.10, 135.77, 142.38, 157.09 ppm. Anal. Calc. for $\mathrm{C}_{27} \mathrm{H}_{47} \mathrm{BrO} \mathrm{C}: 69.36$; $\mathrm{H}: 10.13$. Found: $\mathrm{C}, 69.35 ; \mathrm{H}$, $10.14 \%$.

2-tert-Amyl-1-(2-bromo-ethoxy)-5-pentadecyl-benzene. Colorless oil. Yield: 70\%. FT - IR (neat): 2956, 2922, 2852, 1611, 1503, 1463, 1416, 1247, 1178, 1091, 1023, 817, $721 \mathrm{~cm}^{-1}$. MS $70 \mathrm{eV} \mathrm{m} / \mathrm{z}(\%): 482$ (4) $\left[\mathrm{M}^{+}+2\right], 480$ (4) $\left[\mathrm{M}^{+}\right], 454$ (27), 453 (97), 452 (29), 451 (100), 175 (5), 161 (9), 147 (14), 133 (10), 121 (6), 109 (6), 107 (9), 105 (6), 91 (6), 71 (25), 69 (6), 57 (21), 55 (12). ${ }^{1} \mathrm{H} \mathrm{NMR}\left(\mathrm{CDCl}_{3}, 400 \mathrm{MHz}\right): \delta=0.64(\mathrm{t}, J=7.5 \mathrm{~Hz}, 3 \mathrm{H}), 0.90(\mathrm{t}, J=6.8 \mathrm{~Hz}, 3 \mathrm{H}), 1.25-$ $1.36(\mathrm{~m}, 24 \mathrm{H}), 1.35(\mathrm{~s}, 6 \mathrm{H}), 1.58-1.64(\mathrm{~m}, 2 \mathrm{H}), 1.87(\mathrm{q}, J=7.5 \mathrm{~Hz}, 2 \mathrm{H}), 2.57(\mathrm{t}, J=7.8 \mathrm{~Hz}$, $2 \mathrm{H}), 3.71(\mathrm{t}, J=6.1 \mathrm{~Hz}, 2 \mathrm{H}), 4.33(\mathrm{t}, J=6.1 \mathrm{~Hz}, 2 \mathrm{H}), 6.62(\mathrm{~d}, J=1 \mathrm{~Hz}, 1 \mathrm{H}), 6.75\left(\mathrm{dd}, J_{1}=7.9\right.$ $\left.\mathrm{Hz}, J_{2}=1 \mathrm{~Hz}, 1 \mathrm{H}\right), 7.14(\mathrm{~d}, J=7.9 \mathrm{~Hz}, 1 \mathrm{H}) \mathrm{ppm} .{ }^{13} \mathrm{C} \mathrm{NMR}\left(\mathrm{CDCl}_{3}, 400 \mathrm{MHz}\right): \delta=9.47,13.98$, $22.56,27.99,29.23,29.35,29.41,29.49,29.53,29.56,29.57,31.24,31.80,33.35,35.52$, 38.05, 
67.53, 111.80, 120.45, 127.90, 133.52, 141.73, 156.55 ppm. Anal. Calc. for $\mathrm{C}_{28} \mathrm{H}_{49} \mathrm{BrO}$ C: 69.83; H: 10.26. Found: C, 69.85; H, $10.24 \%$.

\section{Synthesis of compounds 5a-5d. General procedure}

Compound 3a-3d $(7.33 \mathrm{mmol})$ were dissolved in acetone $(15 \mathrm{ml})$; then 4-hydroxybenzaldehyde (4) (1.33 g, $10.90 \mathrm{mmol})$ and potassium carbonate $(3.04 \mathrm{~g}, 22.03 \mathrm{mmol})$ were added. The mixture was stirred under reflux for $24 \mathrm{~h}$. The mixture was cooled to room temperature and filtered to remove the colourless solid formed. The solvent was evaporated under reduced pressure and the crude material was purified by chromatography on silica, eluting with $\mathrm{Et}_{2} \mathrm{O} /$ petroleum ether (3:7), to obtain 5a-d in 40-60\% yields.

4-[2-(3-Pentedec-8-enyl-phenoxy)-ethoxy]-benzaldehyde. White sticky solid. Yield: 60\%. FTIR (neat): 2925, 2853, 2737, 1696, 1601, 1579, 1509, 1486, 1449, 1376, 1312, 1251, 1158, 1110 , 1066, 946, 913, $832 \mathrm{~cm}^{-1}$. MS (EI, $\left.70 \mathrm{eV}\right): \mathrm{m} / \mathrm{z}(\%)=450$ (6), 448 (44), 256 (12), 147 (30), 121 (74), 91 (89), 67 (100). ${ }^{1} \mathrm{H} \mathrm{NMR}\left(\mathrm{CDCl}_{3}, 400 \mathrm{MHz}\right): \delta=0.92$ (t, $\left.J=6.7 \mathrm{~Hz}, 3 \mathrm{H}\right), 1.27-1.33(\mathrm{~m}$, $16 \mathrm{H}), 1.61-1.63(\mathrm{~m}, 2 \mathrm{H}), 2.03-2.06(\mathrm{~m}, 4 \mathrm{H}), 2.60(\mathrm{t}, J=7.7 \mathrm{~Hz}, 2 \mathrm{H}), 4.38(\mathrm{t}, J=5.2 \mathrm{~Hz}, 2 \mathrm{H})$, $4.43(\mathrm{t}, J=4.8 \mathrm{~Hz}, 2 \mathrm{H}), 5.36-5.38(\mathrm{~m}, 2 \mathrm{H}), 6.78-6.85(\mathrm{~m}, 3 \mathrm{H}), 7.09(\mathrm{~d}, J=8.7 \mathrm{~Hz}, 1 \mathrm{H}), 7.23$ $(\mathrm{t}, J=7.6 \mathrm{~Hz}, 1 \mathrm{H}), 7.88(\mathrm{~d}, J=8.8 \mathrm{~Hz}, 2 \mathrm{H}), 9.92(\mathrm{~s}, 1 \mathrm{H}) \mathrm{ppm} .{ }^{13} \mathrm{C} \mathrm{NMR}\left(\mathrm{CDCl}_{3}, 400 \mathrm{MHz}\right): \delta$ $=14.52,23.07,27.60,28.85,29.40,30.03,30.06,31.93,32.20,36.3866 .49,67.30,111.90$, 115.12 , 121.93, 129.67, 130.22, 130.38, 130.64, 132.40, 145.19, 158.86, 164.10, 191.22 ppm. Anal. Calc. for $\mathrm{C}_{30} \mathrm{H}_{42} \mathrm{O}_{3}$ C: 79.96; H: 9.39. Found: C, 79.99; H, 9.40 \%.

4-[2-(3-Pentedecylphenoxy)-ethoxy]-benzaldehyde. Colourless powder. Yield: 57\%. M.p. $72^{\circ} \mathrm{C}$. FT-IR (neat): 2952, 2918, 2848, 1681, 1606, 1582, 1463, 1247, 1167, 1066, 929, $838 \mathrm{~cm}^{-1}$. MS (EI, $70 \mathrm{eV}): \mathrm{m} / \mathrm{z}(\%)=452(45)\left[\mathrm{M}^{+}\right], 256$ (16), 149 (42), 135 (59), 121 (67), 108 (100). ${ }^{1} \mathrm{H}$ NMR $\left(\mathrm{CDCl}_{3}, 400 \mathrm{MHz}\right): \delta=0.84(\mathrm{t}, J=6.8 \mathrm{~Hz}, 3 \mathrm{H}), 1.20-1.25(\mathrm{~m}, 24 \mathrm{H}), 1.54-1.59(\mathrm{~m}, 2 \mathrm{H})$, $2.53(\mathrm{t}, J=7.6 \mathrm{~Hz}, 2 \mathrm{H}), 4.29-4.38(\mathrm{~m}, 4 \mathrm{H}), 6.71-6.78(\mathrm{~m}, 3 \mathrm{H}), 7.01(\mathrm{~d}, J=8.7 \mathrm{~Hz}, 2 \mathrm{H}), 7.16$ $(\mathrm{t}, J=7.7 \mathrm{~Hz}, 1 \mathrm{H}), 7.80(\mathrm{~d}, J=8.7 \mathrm{~Hz}, 2 \mathrm{H}), 9.85$ (s, $1 \mathrm{H}) \mathrm{ppm} .{ }^{13} \mathrm{C} \mathrm{NMR}\left(\mathrm{CDCl}_{3}, 400 \mathrm{MHz}\right): \delta$ $=14.55,23.11,28.90,29.76,29.79,29.94,30.02,30.08,30.10,30.12,31.81,32.35$, 66.49, 67.30, 111.90, 115.33, 121.93, 129.67, 130.62, 132.40, 145.24, 158.84, 164.09, $191.22 \mathrm{ppm}$.

Anal. Calc. for $\mathrm{C}_{30} \mathrm{H}_{44} \mathrm{O}_{3}$ C: 79.60; H: 9.80. Found: C, 79.61; H, $9.79 \%$.

4-[2-(2-tert-Butyl-5-pentadecyl-phenoxy)-ethoxy]-benzaldheyde. Yellow solid. Yield: 48\%. M.p. $44-47^{\circ}$ C. FT-IR (neat): 2922, 2852, 2734, 1697, 1601, 1507, 1417, 1248, 1159, 1091, 984, 931, $830 \mathrm{~cm}^{-1}$. MS (EI, $\left.70 \mathrm{eV}\right): \mathrm{m} / \mathrm{z}(\%)=509(15)\left[\mathrm{M}^{+}+1\right], 508(39)\left[\mathrm{M}^{+}\right], 494(17), 493(52)$, 161 (13), 149 (25), 147 (35), 145 (16), 135 (13), 133 (28), 131 (18), 123 (16), 121 (39), 119 (20), 107 (17), 105 (27), 93 (14), 91 (28), 77 (26), 71 (17), 69 (13), 57 (100), 55 (39). ${ }^{1} \mathrm{H}$ NMR $\left(\mathrm{CDCl}_{3}, 400 \mathrm{MHz}\right): \delta=0.92(\mathrm{t}, J=6.8 \mathrm{~Hz}, 3 \mathrm{H}), 1.28-1.39(\mathrm{~m}, 24 \mathrm{H}), 1.40(\mathrm{~s}, 9 \mathrm{H}), 1.63(\mathrm{qn}, J=$ $7.7 \mathrm{~Hz}, 2 \mathrm{H}), 2.60$ (t, $J=7.7 \mathrm{~Hz}, 2 \mathrm{H}), 4.40-4.52(\mathrm{~m}, 4 \mathrm{H}), 6.76$ (d, J=1.0 Hz, $1 \mathrm{H}), 6.78$ (dd, J= $7.9 \mathrm{~Hz}, J=1.0 \mathrm{~Hz}, 1 \mathrm{H}), 7.08(\mathrm{~d}, J=8.8 \mathrm{~Hz}, 2 \mathrm{H}), 7.23(\mathrm{~d}, J=7.9 \mathrm{~Hz}, 1 \mathrm{H}), 7.89$ (d, $J=8.8 \mathrm{~Hz}$, $2 \mathrm{H}), 9.94(\mathrm{~s}, 1 \mathrm{H}) .{ }^{13} \mathrm{C} \mathrm{NMR}\left(\mathrm{CDCl}_{3}, 400 \mathrm{MHz}\right): \delta=14.56,23.13,29.66,29.78,29.80,29.93$, 29.99, 30.06, 30.10, 30.13, 30.29, 31.89, 32.36, 34.97, 36.11, 66.27, 67.31, 112.78, 115.28, 
121.02, 127.04, 130.63, 132.46, 135.94, 142.35, 157.48, 164.10, 191.18 ppm. Anal. Calc. for $\mathrm{C}_{34} \mathrm{H}_{52} \mathrm{O}_{3} \mathrm{C}: 80.26$; $\mathrm{H}: 10.30$. Found: $\mathrm{C}, 80.26 ; \mathrm{H}, 10.29 \%$.

4-[2-(2-tert-Amyl-5-pentadecyl-phenoxy)-ethoxy]-benzaldehyde. Yellow solid. Yield: 40\%. Mp: $38-41{ }^{\circ} \mathrm{C}$. FT-IR (neat): 2923, 2852, 1697, 1601, 1508, 1417, 1247, 1160, 1092, 1064, $831,722 \mathrm{~cm}^{-1}$. GC-MS (EI, $\left.70 \mathrm{eV}\right): \mathrm{m} / \mathrm{z}(\%)=523(3)\left[\mathrm{M}^{+}+1\right], 522(2)\left[\mathrm{M}^{+}\right], 494(36), 494$ (100), 161 (7), 159 (5), 149 (13), 147 (19), 145 (7), 135 (6), 133 (13), 131 (9), 123 (9), 121 (19), 119 (10), 107 (9), 105 (14), 93 (6), 91 (12), 77 (11), 71 (21), 69 (6), 57 (25), 55 (15). ${ }^{1} \mathrm{H}-\mathrm{NMR}$ $\left(\mathrm{CDCl}_{3}, 400 \mathrm{MHz}\right): \delta=0.63(\mathrm{t}, J=7.5 \mathrm{~Hz}, 3 \mathrm{H}), 0.90(\mathrm{t}, J=6.8 \mathrm{~Hz}, 3 \mathrm{H}), 1.25-1.36(\mathrm{~m}, 24 \mathrm{H})$, 1.33 (s, $6 \mathrm{H}), 1.57-1.66(\mathrm{~m}, 2 \mathrm{H}), 1.83(\mathrm{q}, J=7.5 \mathrm{~Hz}, 2 \mathrm{H}), 2.59$ (t, $J=7.8 \mathrm{~Hz}, 2 \mathrm{H}), 4.37-4.48$ (m, $4 \mathrm{H}), 6.72-6.78(\mathrm{~m}, 2 \mathrm{H}), 7.07(\mathrm{~d}, J=8.7 \mathrm{~Hz}, 2 \mathrm{H}), 7.15(\mathrm{~d}, J=7.8 \mathrm{~Hz}, 1 \mathrm{H}), 7.88(\mathrm{~d}, J=8.7$ $\mathrm{Hz}, 2 \mathrm{H}), 9.93(\mathrm{~s}, 1 \mathrm{H}) \mathrm{ppm} .{ }^{13} \mathrm{C}-\mathrm{NMR}\left(\mathrm{CDCl}_{3}, 400 \mathrm{MHz}\right): \delta=9.56,14.05,22.62,27.94,29.30$, $29.43,29.49,29.56,29.60,29.63,29.64,29.65,31.32,31.86,33.27,35.60,38.06,65.87$, 66.82, $112.17,114.76,120.50,127.89,130.16,131.91,133.71,141.73,156.98,163.59,190.53$.

Anal. Calc. for $\mathrm{C}_{35} \mathrm{H}_{54} \mathrm{O}_{3} \mathrm{C}: 80.41 ; \mathrm{H}: 10.41$. Found: C, 80.42; H, $10.39 \%$.

Synthesis of 5e. Compound $5 a(0.2 \mathrm{~g}, 0.44 \mathrm{mmol})$ was dissolved in dichloromethane $(20 \mathrm{ml})$; then m-chloroperbenzoic acid $(0.14 \mathrm{~g}, 0.81 \mathrm{mmol})$ was added and the mixture was stirred for $3 \mathrm{~h}$ at $0^{\circ} \mathrm{C}$. The mixture was reported to room temperature and washed with $\mathrm{Na}_{2} \mathrm{CO}_{3} 0.25 \mathrm{M}$ to remove the acid formed. Then the mixture was dried and filtered and the solvent was evaporated under reduced pressure, to obtain $5 \mathbf{e}$ in almost quantitative yield.

4-(2-\{3-[7-(3-Hexyl-oxiranyl)-heptyl]-phenoxy\}-ethoxy)-benzaldehyde white sticky solid. Yield: 70 \%. FT-IR (neat): 2925, 2854, 1696, 1600, 1579, 1508, 1486, 1449, 1252, 1190, 1158 , 1066, 930, $832 \mathrm{~cm}^{-1}$. MS (EI, $\left.70 \mathrm{eV}\right): \mathrm{m} / \mathrm{z}(\%)=466$ (21), 448 (21), 409 (13), 339 (20), 268 (24), 256 (23), 149 (39), 133 (40), 121 (100), 107 (61), 91 (88), 77 (83), 55 (88). ${ }^{1} \mathrm{H}$ NMR $\left(\mathrm{CDCl}_{3}, 400 \mathrm{MHz}\right): \delta=0.91(\mathrm{t}, J=6.8 \mathrm{~Hz}, 3 \mathrm{H}), 1.28-1.36(\mathrm{~m}, 16 \mathrm{H}), 1.60-1.63(\mathrm{~m}, 2 \mathrm{H}), 2.60(\mathrm{t}$, $J=7.7 \mathrm{~Hz}, 2 \mathrm{H}), 2.92(\mathrm{~m}, 4 \mathrm{H}), 4.37(\mathrm{t}, J=5.2 \mathrm{~Hz}, 2 \mathrm{H}), 4.43(\mathrm{t}, J=4.8 \mathrm{~Hz}, 2 \mathrm{H}), 6.77-6.84(\mathrm{~m}$, $3 \mathrm{H}), 7.09$ (d, $J=4.4 \mathrm{~Hz}, 2 \mathrm{H}), 7.22$ (t, $J=3.7 \mathrm{~Hz}, 1 \mathrm{H}), 7.87$ (d, $J=5.0 \mathrm{~Hz}, 2 \mathrm{H}), 9.92$ (s, $1 \mathrm{H})$ ppm. ${ }^{13} \mathrm{C} \mathrm{NMR}\left(\mathrm{CDCl}_{3}, 400 \mathrm{MHz}\right): 14.50,22.98,26.98,27.03,28.21,28.24,29.60,29.63$, 31.72 , 32.18, 36.38, 57.66, 57.67, 66.51, 67.49, 111.94, 115.38, 121.91, 129.69, 130.62, 132.86, 145.09, 158.88, 164.11, 191.22 ppm. Anal. Calc. for $\mathrm{C}_{30} \mathrm{H}_{42} \mathrm{O}_{4} \mathrm{C}: 77.21$; H: 9.07. Found: C, $77.22 ; \mathrm{H}, 9.05 \%$.

Synthesis of $5 f$. C627 (15.60 mg, $0.025 \mathrm{mmol}$ ) was added under $\mathrm{N}_{2}$ atmosphere to a solution of 5a $(559.7 \mathrm{mg}, 1.2 \mathrm{mmol})$ in $6 \mathrm{~mL}$ of dichloromethane, producing a light green solution which were stirred at reflux for $45 \mathrm{~h}$. The mixture was then concentrated in vacuo to a dark brown oil. The crude material was purified by silica gel chromatography (dichloromethane) affords compound $5 f$ as a white solid (183.9 $\mathrm{mg}, 42 \%)$.

Representative for the compound 5f. 4,4'-(2,2'-(3,3'-(Hexadec-8-ene-1,16-diyl)bis(3,1phenylene))bis(oxy)bis(ethane-2,1-diyl))bis(oxy)dibenzaldehyde. Yield: $42 \%$. Mp 69-72 ${ }^{\circ} \mathrm{C}$. FTIR (neat): 2992, 2919, 2848, 1770, 1759, 1680, 1605, 1509, 1454, 1376, 1307, 1275, 1246, $1167,1065,962,929,858,837 \mathrm{~cm}^{-1}$. MS (EI, $\left.70 \mathrm{eV}\right): \mathrm{m} / \mathrm{z}(\%)=705[\mathrm{M}+1]^{+}$. ${ }^{1} \mathrm{H}$ NMR $(400$ $\left.\mathrm{MHz}, \mathrm{CDCl}_{3}\right): \delta=1.29-1.31(\mathrm{~m}, 16 \mathrm{H}), 1.58-1.61(\mathrm{~m}, 4 \mathrm{H}), 1.96-2.01(\mathrm{~m}, 4 \mathrm{H}), 2.57(\mathrm{t}, 4 \mathrm{H}, \mathrm{J}=$ 
$8.0 \mathrm{~Hz}), 4.34(\mathrm{t}, 4 \mathrm{H}, \mathrm{J}=4.8 \mathrm{~Hz}), 4.41(\mathrm{t}, 4 \mathrm{H}, \mathrm{J}=4.8 \mathrm{~Hz}), 5.38(\mathrm{t}, 2 \mathrm{H}, \mathrm{J}=3.2 \mathrm{~Hz}), 6.76(\mathrm{~d}, 2 \mathrm{H}, \mathrm{J}$ $=8.0 \mathrm{~Hz}), 6.78(\mathrm{~s}, 2 \mathrm{H}), 6.81(\mathrm{~d}, 2 \mathrm{H}), 7.04(\mathrm{~d}, 2 \mathrm{H}, \mathrm{J}=8.0 \mathrm{~Hz}), 7.19(\mathrm{t}, 2 \mathrm{H}, \mathrm{J}=8.4 \mathrm{~Hz}), 7.83(\mathrm{~d}$, $2 \mathrm{H}, \mathrm{J}=8.4 \mathrm{~Hz}$ ), 9.88 (s, 2H). ${ }^{13} \mathrm{C}$ NMR (100 MHz, CDCl3): d 29.1, 29.2, 29.3, 29.6, 31.3, 32.5, 35.9, 66.0, 66.8, 111.4, 114.8, 121.4, 129.2, 129.8, 130.1, 130.3, 131.9, 144.7, 158.4, 163.6, 190.7. Anal. Calc. for $\mathrm{C}_{46} \mathrm{H}_{56} \mathrm{O}_{6}$ : C, 78.41; H, 7.95. Found: C, 78.18; H, 7.72\%.

\section{General procedure for the synthesis of compounds 7a-i}

Compound 5a-e $(0,2 \mathrm{mmol})$, fullerene $\left[\mathrm{C}_{60}\right](0.144 \mathrm{~g}, 0.2 \mathrm{mmol})$ and $N$-methylglicine 6a (or $N$ phenylglicine $6 \mathbf{b})(0.2 \mathrm{mmol})$ were reacted in refluxing toluene $(500 \mathrm{ml})$ under nitrogen atmosphere for $24 \mathrm{~h}$. The mixture was cooled to room temperature and the resulting solution was evaporated to dryness. The crude product was purified by chromatography on a silica gel column, eluting with toluene to obtain compound $\mathbf{7 a - h}$ in $28-35 \%$ yield. A similar procedure was accomplished for the synthesis of the compound $7 \mathbf{j}$ starting from $5 \mathbf{f}(0,1 \mathrm{mmol})$, fullerene $\left[\mathrm{C}_{60}\right](0.144 \mathrm{~g}, 0.2 \mathrm{mmol})$ and $N$-methylglicine $6 \mathbf{6 a}(0.2 \mathrm{mmol})$ using the previously reported procedure.

Synthesis of $7 \mathbf{j}$ via metathesis reaction of $7 \mathbf{i} .40 \mathrm{mg}(0.033 \mathrm{mmol})$ of fulleropyrrolidine $7 \mathbf{i}$ were dissolved in $21 \mathrm{ml}$ of dichloromethane. Then, a solution of the catalyst C627 (0.42 $\mathrm{mg}$ in $1.5 \mathrm{ml}$ of $\mathrm{CH}_{2} \mathrm{Cl}_{2}, 0.02$ eq.) was added and the mixture refluxed with stirring for $44 \mathrm{~h}$. The crude product was purified with silica gel using toluene as eluent. Compound $\mathbf{7 j}$ (brown solid) was isolated in $30 \%$ yield.

Representative data for compounds 7a-7j. Compound 7a. $N$-Methyl-2-[4-(2-(3-pentadecen8-yl-phenoxy)-ethoxy)-phenyl]-fulleropyrrolidine brown sticky solid at room temperature. Yield: $33 \% \mathrm{mp} 26-36^{\circ} \mathrm{C}$. FT-IR (neat): 2922, 2851, 2781, 1609, 1583, 1511, 1463, 1377, 1247 , 1172, 1159, 1072, 888, 832, 721. LC-MS (ESI): $\mathrm{m} / \mathrm{z}=1198[\mathrm{M}+\mathrm{H}]^{+} .{ }^{1} \mathrm{H} \mathrm{NMR}\left(\mathrm{CDCl}_{3}, 400\right.$ MHz): $\delta=0.91(\mathrm{t}, J=9.5 \mathrm{~Hz}, 3 \mathrm{H}), 1.27-1.37(\mathrm{~m}, 16 \mathrm{H}), 2.05-2.07(\mathrm{~m}, 4 \mathrm{H}), 2.58-2.61(\mathrm{t}, J=7.7$ $\mathrm{Hz}, 2 \mathrm{H}), 2.82(\mathrm{~s}, 3 \mathrm{H}), 4.27(\mathrm{~d}, J=9.4 \mathrm{~Hz}, 1 \mathrm{H}), 4.35(\mathrm{~m}, 2 \mathrm{H}), 4.73(\mathrm{~m}, 2 \mathrm{H}), 4.92(\mathrm{~s}, 1 \mathrm{H}), 5.00$ $(\mathrm{d}, J=9.3 \mathrm{~Hz}, 1 \mathrm{H}), 5.38-5.39(\mathrm{~m}, 2 \mathrm{H}), 6.77-6.82(\mathrm{~m}, 3 \mathrm{H}), 7.03(\mathrm{t}, J=7.2 \mathrm{~Hz}, 2 \mathrm{H}), 7.21(\mathrm{t}, J=$ $8.0 \mathrm{~Hz}, 1 \mathrm{H}), 7.75(\mathrm{~m}$, broad signal, $2 \mathrm{H}) \mathrm{ppm} .{ }^{13} \mathrm{C} \mathrm{NMR}\left(\mathrm{CDCl}_{3}, 400 \mathrm{MHz}\right): 14.55,23.13$, 23.61, 29.41, 29.66, 29.81, 30.61, 32.37, 36.72, 37.82, 39.27, 40.39, 44.72, 66.75, 66.91, 69.40, $70.44,83.60,107.70,111.92,115.39,121.73,125.94,129.60,129.80,130.24,130.36,130.92$, $136.22,136.98,137.22,140.59,142.45,142.55,142.71,143.00,144.82,145.07,145.66,145.89$, 145.96, 146.37, 146.57, 146.75, 146.93, 147.73, 154.04, 154.51, 156.78, 159.02, 159.13. Anal. Calc. for $\mathrm{C}_{92} \mathrm{H}_{47} \mathrm{NO}_{2}$ : C, 92.21; H, 3.95; N, 1.17. Found: C, 92.23; H, 3.91; N, $1.17 \%$.

$\mathrm{N}$-Methyl-2-[4-(2-(3-pentadecyl-phenoxy)-ethoxy)-phenyl]-fulleropyrrolidine (7b). Brown solid; mp: $189-191{ }^{\circ} \mathrm{C}$. Yield: 35\% FT-IR (neat): 2920, 2849, 2779, 1608, 1583, 1510, 1462, 1258, 1246, 1171, 1159, 1089, 1015, $796 \mathrm{~cm}^{-1}$. MS (ESI): m/z (\%) = $1200[\mathrm{M}+\mathrm{H}]^{+} .{ }^{1} \mathrm{H}-\mathrm{NMR}$ $\left(\mathrm{CDCl}_{3}, 400 \mathrm{MHz}\right): \delta=0.90(\mathrm{t}, J=6.8 \mathrm{~Hz}, 3 \mathrm{H}), 1.23-1.35(\mathrm{~m}, 24 \mathrm{H}), 1.55-1.63(\mathrm{~m}, 2 \mathrm{H}), 2.58$ $(\mathrm{t}, J=7.7 \mathrm{~Hz}, 3 \mathrm{H}), 2.58(\mathrm{t}, J=7.7 \mathrm{~Hz}, 2 \mathrm{H}), 2.82(\mathrm{~s}, 3 \mathrm{H}), 4.26(\mathrm{~d}, J=9.4 \mathrm{~Hz}, 1 \mathrm{H}), 4.32-4.36$ $(\mathrm{m}, 4 \mathrm{H}), 4.91(\mathrm{~s}, 1 \mathrm{H}), 5.00(\mathrm{~d}, J=9.4 \mathrm{~Hz}, 1 \mathrm{H}), 6.75-6.82(\mathrm{~m}, 3 \mathrm{H}), 7.03(\mathrm{~d}, J=8.1 \mathrm{~Hz}, 2 \mathrm{H})$, $7.20(\mathrm{t}, J=7.8 \mathrm{~Hz}, 1 \mathrm{H}), 7.70-7.80$ (m, broad signal, $2 \mathrm{H})$ ppm. ${ }^{13} \mathrm{C}-\mathrm{NMR}\left(\mathrm{CDCl}_{3}, 400 \mathrm{MHz}\right): \delta$ 
$=14.11,22.67,23.78,29.33,29.49,29.66,30.37,31.34,31.90,35.98,39.94,66.31,66.47$, $69.31,69.97,77.79,83.15,111.49,114.77,114.93,121.29,129.15,129.34,130.50,135.72$, $136.51,136.76,138.05,139.57,140.12,141.51,141.65,141.93,142.09,142.26,142.52,142.65$, $142.95,143.10,144.36,144.59,144.69,145.20,145.45,145.90,146.10,146.30,146.48,147.27$, 153.60, 154.07, 156.34, 158.55, 158.66 ppm. Anal. Calc. for $\mathrm{C}_{92} \mathrm{H}_{49} \mathrm{NO}_{2}: \mathrm{C}, 92.05 ; \mathrm{H}, 4.11 ; \mathrm{N}$, 1.17. Found: C, 92.08; H, 3.94; N, $1.18 \%$.

N-Methyl-2-[4-(2-(2-tert-butyl-5-pentadecyil-phenoxy)-ethoxy)-phenyl]-fulleropyrrolidine

(7c). Brown solid; mp: $151-153^{\circ} \mathrm{C}$. Yield: 30\% FT-IR (neat): 2908, 2850, 2779, 1609, 1509, 1460, 1416, 1332, 1243, 1174, 1090, 841, 830, $705 \mathrm{~cm}^{-1}$. MS (ESI): m/z (\%) = $1256[\mathrm{M}+\mathrm{H}]^{+}$. ${ }^{1} \mathrm{H}-\mathrm{NMR}\left(\mathrm{CDCl}_{3}, 400 \mathrm{MHz}\right): \delta=0.91(\mathrm{t}, J=6.8 \mathrm{~Hz}, 3 \mathrm{H}), 1.24-1.37(\mathrm{~m}, 24 \mathrm{H}), 1.37(\mathrm{~s}, 9 \mathrm{H})$, 1.56-1.64 (m, $2 \mathrm{H}), 2.58(\mathrm{t}, J=7.7 \mathrm{~Hz}, 2 \mathrm{H}), 2.83(\mathrm{~s}, 3 \mathrm{H}), 4.27(\mathrm{~d}, J=9.4 \mathrm{~Hz}, 1 \mathrm{H}), 4.32-4.36$ $(\mathrm{m}, 4 \mathrm{H}), 4.92(\mathrm{~s}, 1 \mathrm{H}), 5.00(\mathrm{~d}, J=9.4 \mathrm{~Hz}, 1 \mathrm{H}), 6.72-6.76(\mathrm{~m}, 2 \mathrm{H}), 7.02(\mathrm{~d}, J=8.6 \mathrm{~Hz}, 2 \mathrm{H})$, $7.19(\mathrm{~d}, J=7.7 \mathrm{~Hz}, 1 \mathrm{H}), 7.70-7.80(\mathrm{~m}$, broad signal, $2 \mathrm{H}) \mathrm{ppm} .{ }^{13} \mathrm{C}-\mathrm{NMR}\left(\mathrm{CDCl}_{3}, 400 \mathrm{MHz}\right): \delta$ $=14.09,22.66,29.32,29.48,29.52,29.59,29.62,29.66,29.88,31.38,31.89,34.49,35.64$, $39.96,66.17,66.50,68.94,69.97,77.32,83.11,112.41,113.90,120.39,126.47,129.28,135.52$, $135.72,135.77,136.52,136.74,138.07,139.55,139.86,140.09,141.49,141.64,141.81,141.84$, $141.92,141.97,142.00,142.05,142.07,142.09,142.12,142.22,142.25,142.52,142.54,142.64$, $142.95,143.10,144.36,144.58,144.67,145.11,145.20,145.24,145.28,145.30,145.43,145.45$, $145.51,145.74,145.91,146.06,146.12,146.17,146.23,146.27,146.30,146.48,146.76,147.27$, 153.59, 154.08, 156.34, 157.16, 158.69 ppm. Anal. Calc. for $\mathrm{C}_{96} \mathrm{H}_{57} \mathrm{NO}_{2}: \mathrm{C}, 91.77 ; \mathrm{H}, 4.57 ; \mathrm{N}$, 1.11. Found: C, 91.79; H, 4.54; N, $1.12 \%$.

N-Methyl-2-[4-(2-(2-tert-amyl-5-pentadecyl-phenoxy)-ethoxy)-phenyl]-fulleropyrrolidine

(7d). Brown solid; mp: 196-198 ${ }^{\circ} \mathrm{C}$. Yield: 28\% FT-IR (neat) 3034, 2921, 2850, 2779, 1509, 1461, 1416, 1332, 1295, 1243, 1175, 1092, 907, 830, $736 \mathrm{~cm}^{-1}$. MS (ESI): $\mathrm{m} / \mathrm{z}(\%)=1270$ $[\mathrm{M}+\mathrm{H}]^{+} .{ }^{1} \mathrm{H} \mathrm{NMR}\left(\mathrm{CDCl}_{3}, 400 \mathrm{MHz}\right): \delta=0.61(\mathrm{t}, J=7.5 \mathrm{~Hz}, 3 \mathrm{H}), 0.91(\mathrm{t}, J=6.8 \mathrm{~Hz}, 3 \mathrm{H})$, 1.25-1.33 (m, $30 \mathrm{H}), 1.59-1.64(\mathrm{~m}, 2 \mathrm{H}), 1.83(\mathrm{q}, J=7.5 \mathrm{~Hz}, 2 \mathrm{H}), 2.57(\mathrm{t}, J=7.8 \mathrm{~Hz}, 2 \mathrm{H}), 2.83$ $(\mathrm{s}, 3 \mathrm{H}), 4.27(\mathrm{~d}, J=9.4 \mathrm{~Hz}, 1 \mathrm{H}), 4.30-4.41(\mathrm{~m}, 4 \mathrm{H}), 5.00(\mathrm{~d}, J=9.4 \mathrm{~Hz}, 1 \mathrm{H}), 6.70-6.76$ (m, 2 $\mathrm{H}), 7.02(\mathrm{~d}, J=8.4 \mathrm{~Hz}, 2 \mathrm{H}), 7.12(\mathrm{~d}, J=7.8 \mathrm{~Hz}, 1 \mathrm{H}), 7.70-7.78(\mathrm{~m}$, broad signal, $2 \mathrm{H}) \mathrm{ppm} .{ }^{13} \mathrm{C}$ NMR $\left(\mathrm{CDCl}_{3}, 400 \mathrm{MHz}\right): \delta=9.59,9.72,14.11,22.69,27.97,28.02,29.36,29.55,29.69,30.15$, $31.37,31.91,33.29,35.66,38.11,40.00,66.23,66.48,68.95,69.96,77.19,83.09,112.31$, $114.70,120.35,127.84,129.25,130.48,133.77,135.75,135.79,136.54,136.75,139.56,139.88$, $140.10,140.13,141.50,141.66,141.75,141.82,141.93,141.98,142.01,142.08,142.10,142.13$, $142.23,142.26,142.53,142.65,142.96,143.12,144.37,144.59,144.68,145.12,145.21,145.25$, $145.29,145.31,145.45,145.46,145.52,145.75,145.91,146.07,146.11,146.13,146.19,146.25$, $146.29,146.50,146.77,147.27,153.59,154.08,156.34,157.15,158.69$ ppm. Anal. Calc. for $\mathrm{C}_{97} \mathrm{H}_{59} \mathrm{NO}_{2}$ : C, 91.70; H, 4.68; N, 1.10. Found: C, 91.74; H, 4.66; N, $1.09 \%$.

$\mathbf{N}$-Phenyl-2-[4-(2-(3-pentadecyl-phenoxy)-ethoxy)-phenyl]-fulleropyrrolidine (7e). Brown solid; mp: $120-123^{\circ} \mathrm{C}$. Yield: 29\% FT-IR (neat): 2922, 2850, 1599, 1583, 1509, 1495, 1463 , $1245,1172,1157,1071,1035,832,754,693 \mathrm{~cm}^{-1}$. MS (ESI): $\mathrm{m} / \mathrm{z}(\%)=1262[\mathrm{M}+\mathrm{H}]^{+} .{ }^{1} \mathrm{H}$ NMR $\left(\mathrm{CDCl}_{3}, 400 \mathrm{MHz}\right): \delta=0.91(\mathrm{t}, J=6.8 \mathrm{~Hz}, 3 \mathrm{H}), 1.25-1.36(\mathrm{~m}, 24 \mathrm{H}), 1.56-1.61(\mathrm{~m}, 2 \mathrm{H}), 2.58(\mathrm{t}$, 
$J=7.6 \mathrm{~Hz}, 2 \mathrm{H}), 4.27-4.31(\mathrm{~m}, 4 \mathrm{H}), 5.00(\mathrm{~d}, J=9.9 \mathrm{~Hz}, 1 \mathrm{H}), 5.68(\mathrm{~d}, J=9.9 \mathrm{~Hz}, 1 \mathrm{H}), 6.06(\mathrm{~s}$, $1 \mathrm{H}), 6.72-6.82(\mathrm{~m}, 3 \mathrm{H}), 6.94(\mathrm{~d}, J=8.7 \mathrm{~Hz}, 2 \mathrm{H}), 7.19$ (t, $J=7.8 \mathrm{~Hz}, 1 \mathrm{H}), 7.36-7.40$ (m, $5 \mathrm{H})$, $7.74(\mathrm{~d}, J=8.7 \mathrm{~Hz}, 2 \mathrm{H}) \mathrm{ppm} .{ }^{13} \mathrm{C} \mathrm{NMR}\left(\mathrm{CDCl}_{3}, 400 \mathrm{MHz}\right) \delta: 14.11,22.67,29.33,29.50,29.57$, 29.66, 31.33, 31.90, 35.98, 66.29, 66.40, 68.07, 68.54, 76.14, 76.50, 76.82, 77.14, 111.49, $114.88,114.93,121.27,121.58,122.34,129.11,129.51,129.76,130.17,135.72,136.50,136.56$, $139.50,139.91,140.14,140.24,141.55,141.69,141.83,141.91,142.04,142.10,142.14,142.23$, $142.28,142.58,142.68,143.01,143.07,143.15,144.43,144.62,144.67,145.13,145.27,145.31$, $145.44,145.56,145.71,145.94,146.10,146.15,146.21,146.27,146.35,146.62,147.35,153.42$, 153.58, 153.76, 155.99, 158.33, 158.55 ppm. Anal. Calc. for $\mathrm{C}_{97} \mathrm{H}_{51} \mathrm{NO}_{2}: \mathrm{C}, 92.28 ; \mathrm{H}, 4.07 ; \mathrm{N}$, 1.11. Found: C, 92.30; H, 4.04; N, $1.08 \%$.

\section{$\mathrm{N}$-Phenyl-2-[4-(2-(2-tert-butyl-5-pentadecyl-phenoxy)-ethoxy)-phenyl]-fulleropyrrolidine}

(7f). Brown solid; mp: $118-120^{\circ} \mathrm{C}$. Yield: $28 \%$ FT-IR (neat): 2922, 2850, 1599, 1506, 1462, 1417, 1359, 1303, 1244, 1173, 1090, 905, 831, 731, $697 \mathrm{~cm}^{-1}$. MS (ESI): m/z $1318[\mathrm{M}+\mathrm{H}]^{+} .{ }^{1} \mathrm{H}$ NMR $\left(\mathrm{CDCl}_{3}, 400 \mathrm{MHz}\right): \delta=0.90(\mathrm{t}, J=6.8 \mathrm{~Hz}, 3 \mathrm{H}), 1.25-1.34(\mathrm{~m}, 24 \mathrm{H}), 1.34(\mathrm{~s}, 9 \mathrm{H}), 1.57-$ $1.63(\mathrm{~m}, 2 \mathrm{H}), 2.55$ (t, $J=7.8 \mathrm{~Hz}, 2 \mathrm{H}), 4.29-4.36(\mathrm{~m}, 4 \mathrm{H}), 5.01$ (d, $J=9.9 \mathrm{~Hz}, 1 \mathrm{H}), 5.69$ (d, $J=$ $9.9 \mathrm{~Hz}, 1 \mathrm{H}), 6.07(\mathrm{~s}, 1 \mathrm{H}), 6.69-6.75(\mathrm{~m}, 3 \mathrm{H}), 6.93$ (d, J = 8.7 Hz, $2 \mathrm{H}), 7.17$ (d, J = 7.8 Hz, 1 $\mathrm{H}), 7.37-7.41(\mathrm{~m}, 5 \mathrm{H}), 7.74(\mathrm{~d}, J=8.7 \mathrm{~Hz}, 2 \mathrm{H}) \mathrm{ppm} .{ }^{13} \mathrm{C} \mathrm{NMR}\left(\mathrm{CDCl}_{3}, 400 \mathrm{MHz}\right): \delta=14.10$, 22.66, 29.33, 29.52, 29.67, 29.87, 30.01, 31.38, 31.90, 34.47, 35.64, 66.15, 66.43, 68.06, 68.57, $76.10,112.42,114.90,120.38,121.57,122.34,126.46,127.10,129.13,129.75,130.09,135.52$, $135.71,136.54,139.47,139.88,140.13,140.24,141.53,141.69,141.84,142.03,142.13,142.23$, $142.58,143.06,143.15,144.43,144.60,144.68,145.27,145.45,145.55,145.71,145.94,146.09$, $146.14,146.26,146.65,146.92,147.37,153.44,153.57,153.78,156.00,157.15,158.37$ ppm. Anal. Calc. for $\mathrm{C}_{101} \mathrm{H}_{59} \mathrm{NO}_{2}$ : C, 92.00; H, 4.51; N, 1.06. Found: C, 92.03; H, 4.51; N, $1.05 \%$.

\section{$\mathbf{N}$-Phenyl-2-[4-(2-(2-tert-amyl-5-pentadecyl-phenoxy)-ethoxy)-phenyl]-fulleropyrrolidine}

(7g). Brown solid; mp 128-130 ${ }^{\circ}$ C. Yield: 28\%. FT-IR (neat): 2920, 2849, 1598, 1507, 1460, 1416, 1242, 1172, 1092, 830, 759, 736, $694 \mathrm{~cm}^{-1}$. MS (ESI): m/z (\%) = $1332[\mathrm{M}+\mathrm{H}]^{+} .{ }^{1} \mathrm{H} \mathrm{NMR}$ $\left(\mathrm{CDCl}_{3}, 400 \mathrm{MHz}\right): \delta=0.58(\mathrm{t}, J=7.4 \mathrm{~Hz}, 3 \mathrm{H}), 0.90(\mathrm{t}, J=6.8 \mathrm{~Hz}, 3 \mathrm{H}), 1.25-1.33(\mathrm{~m}, 30 \mathrm{H})$, 1.58-1.63 (m, $2 \mathrm{H}), 1.79(\mathrm{q}, J=7.4 \mathrm{~Hz}, 2 \mathrm{H}), 2.55$ (t, $J=7.7 \mathrm{~Hz}, 2 \mathrm{H}), 4.27-4.33(\mathrm{~m}, 4 \mathrm{H}), 5.01$ $(\mathrm{d}, J=9.6 \mathrm{~Hz}, 1 \mathrm{H}), 5.68(\mathrm{~d}, J=9.6 \mathrm{~Hz}, 1 \mathrm{H}), 6.07(\mathrm{~s}, 1 \mathrm{H}), 6.67-6.74(\mathrm{~m}, 3 \mathrm{H}), 6.93(\mathrm{~d}, J=7.6$ $\mathrm{Hz}, 2 \mathrm{H}), 7.10(\mathrm{~d}, J=7.9 \mathrm{~Hz}, 1 \mathrm{H}), 7.38-7.41(\mathrm{~m}, 5 \mathrm{H}), 7.73(\mathrm{~d}, J=7.6 \mathrm{~Hz}, 2 \mathrm{H}) \mathrm{ppm} .{ }^{13} \mathrm{C} \mathrm{NMR}$ $\left(\mathrm{CDCl}_{3}, 400 \mathrm{MHz}\right): \delta=9.57,9.69,14.13,22.69,27.95,29.36,29.54,29.69,30.94,31.36,31.92$, $33.27,35.65,38.10,66.21,66.41,68.08,68.60,76.12,76.87,77.19,112.33,114.88,120.35$, $121.60,122.36,125.28,127.82,128.21,129.03,129.16,129.76,130.08,133.80,135.73,136.48$, $136.56,139.49,139.90,140.15,140.26,141.55,141.71,141.75,141.84,141.91,142.05,142.12$, $142.15,142.25,142.28,142.59,142.70,143.02,143.09,143.17,144.44,144.63,144.70,145.15$, $145.27,145.46,145.57,145.73,145.96,146.11,146.16,146.22,146.28,146.37,146.67,147.39$, $153.45,153.59,153.79,156.02,157.15,158.38$ ppm. Anal. Calc. for $\mathrm{C}_{102} \mathrm{H}_{61} \mathrm{NO}_{2}$ : C, 91.93; $\mathrm{H}$, 4.61; N, 1.05. Found: C, 91.93; H, 4.59; N, $1.07 \%$.

Compound 7h.brown sticky solid. Yield: 33\%. FT-IR (neat): 2923, 2852, 1602, 1583, 1510, 1462, 1377, 1249, 1158, 1072, 963, $909 \mathrm{~cm}^{-}{ }^{1}$. LC-MS (APCI): m/z $=1214[\mathrm{M}+\mathrm{H}]^{+} .{ }^{1} \mathrm{H}$ NMR 
$\left(\mathrm{CDCl}_{3}, 400 \mathrm{MHz}\right): \delta=0.90(\mathrm{t}, J=6.8 \mathrm{~Hz}, 3 \mathrm{H}), 1.28-1.34(\mathrm{~m}, 16 \mathrm{H}), 2.59(\mathrm{t}, J=9.2 \mathrm{~Hz}, 2 \mathrm{H})$, $2.82(\mathrm{~s}, 3 \mathrm{H}), 2.92(\mathrm{~m}, 4 \mathrm{H}), 3.65(\mathrm{t}, J=6.4 \mathrm{~Hz}, 2 \mathrm{H}), 4.26(\mathrm{~d}, J=9.4 \mathrm{~Hz}, 1 \mathrm{H}), 4.30-4.34(\mathrm{~m}, 4$ H), $4.92(\mathrm{~s}, 1 \mathrm{H}), 5.00(\mathrm{~d}, J=4.6 \mathrm{~Hz}, 1 \mathrm{H}), 6.77-6.82(\mathrm{~m}, 3 \mathrm{H}), 7.03(\mathrm{~d}, J=4.2 \mathrm{~Hz}, 2 \mathrm{H}), 7.21(\mathrm{t}$, $J=2.0 \mathrm{~Hz}, 1 \mathrm{H}), 7.70-7.78(\mathrm{~m}$, broad signal, $2 \mathrm{H}) \mathrm{ppm} .{ }^{13} \mathrm{C} \mathrm{NMR}\left(\mathrm{CDCl}_{3}, 400 \mathrm{MHz}\right): \delta=14.62$, 23.22, 30.47, 33.83, 36.70, 37.82, 39.49, 40.62, 44.71, 57.57, 66.51, 66.94, 67.93, 68.20, 70.43, $83.19,112.92,112.95,118.59,121.16,121.69,129.67,130.36,132.38,132.90,140.33,142.43$, $142.45,142.53,142.54,142.70,143.40,145.73,146.34,146.62,146.92,147.72,154.01,154.05$, $154.51,156.79,158.52,159.04,159.13,168.00$ ppm. Anal. Calc. for $\mathrm{C}_{92} \mathrm{H}_{47} \mathrm{NO}_{3}$ : C, 90.99; $\mathrm{H}$, 3.90; N, 1.15. Found: C, 91.00; H, 3.88; N, $1.11 \%$.

Compound 7i. brown sticky solid. Yield: 30\% FT-IR (neat): 2921, 2850, 2780, 1770, 1759, $1680,1605,1509,1458,1376,1306,1275,1246,1169,1065,962,929,858,837 \mathrm{~cm}^{-1}$. LC-MS (APCI): $\mathrm{m} / \mathrm{z}=1370[\mathrm{M}+\mathrm{H}]^{+} .{ }^{1} \mathrm{H}$ NMR $\left(400 \mathrm{MHz}, \mathrm{CDCl}_{3}\right): \delta=1.28-1.32(\mathrm{~m}, 16 \mathrm{H}), 1.58-1.61$ $(\mathrm{m}, 4 \mathrm{H}), 1.96-2.01(\mathrm{~m}, 4 \mathrm{H}), 2.57(\mathrm{t}, J=7.8 \mathrm{~Hz}, 4 \mathrm{H}), 2.81(\mathrm{~s}, 3 \mathrm{H}), 4.22-4.36(\mathrm{~m}, 5 \mathrm{H}), 4.38-$ $4.44(\mathrm{~m}, 4 \mathrm{H}), 4.91(\mathrm{~s}, 1 \mathrm{H}), 4.99(\mathrm{~d}, J=9.4 \mathrm{~Hz}, 1 \mathrm{H}), 5.36-5.40(\mathrm{~m}, 2 \mathrm{H}), 6.76-6.84(\mathrm{~m}, 6 \mathrm{H})$, 7.05 (d, $J=8.7 \mathrm{~Hz}, 4 \mathrm{H}), 7.22$ (t, $J=8.4 \mathrm{~Hz}, 2 \mathrm{H}), 7.87$ (d, $J=8.7 \mathrm{~Hz}, 4 \mathrm{H}), 9.91$ (s, $1 \mathrm{H}) \mathrm{ppm}$.

${ }^{13} \mathrm{C} \mathrm{NMR}\left(\mathrm{CDCl}_{3}, 400 \mathrm{MHz}\right): \delta=11.38,14.48,23.41,24.15,29.33,29.50,29.71,29.78,30.76$, $31.78,33.00,36.41,39.13,66.48,67.29,68.56,111.88,115.33,121.92,129.21,130.62,130.75$, $131.30,132.41,132.85,136.21,140.53,142.56,144.80,145.63,145.89,146.18,146.62,147.70$, 158.84, 164.08, 168.18, 191.21 ppm. Anal. Calc. for $\mathrm{C}_{101} \mathrm{H}_{47} \mathrm{NO}_{6}: \mathrm{C}, 88.52 ; \mathrm{H}, 3.46 ; \mathrm{N}, 1.02$. Found: C, 88.56; H, 3.44; N, $1.00 \%$.

Compound 7j.Brown solid. Yield: 30\% . Mp 186-191 ${ }^{\circ} \mathrm{C} .2921,2850,2781,1609,1582,1510$, 1463, 1377, 1247, 1172, 1159, 1072, 888, 832, $721 \mathrm{~cm}^{-1} .{ }^{1} \mathrm{H}$ NMR $\left(400 \mathrm{MHz}, \mathrm{CDCl}_{3}\right): \delta=1.13-$ $1.38\left(\mathrm{~m}, \mathrm{CH}_{2}, 16 \mathrm{H}\right), 1.59(\mathrm{~m}, 4 \mathrm{H}), 1.98(\mathrm{~m}, 4 \mathrm{H}), 2.59$ (t, $\left.J=9.1 \mathrm{~Hz}, 4 \mathrm{H}\right), 4.24(\mathrm{~d}, J=5.7 \mathrm{~Hz}$, $2 \mathrm{H}), 2.81(\mathrm{~s}, 6 \mathrm{H}), 4.91(\mathrm{~s}, 2 \mathrm{H}), 4.33(\mathrm{~m}, 8 \mathrm{H}), 4.99$ (d, J=9.2 Hz 2H), $5.38(\mathrm{~m}, 2 \mathrm{H}), 6.80-6.75$ $(\mathrm{m}, 6 \mathrm{H}), 7.02$ (d, $J=8.6 \mathrm{~Hz}, 4 \mathrm{H}), 7.19$ (t, $J=7.9 \mathrm{~Hz}, 2 \mathrm{H}), 7.75$ (m, broad signal, $4 \mathrm{H}) \mathrm{ppm}$. Anal. Calc. for $\mathrm{C}_{156} \mathrm{H}_{38} \mathrm{~N}_{2} \mathrm{O}_{6}$ : C, 92.03; H, 1.88; N, 1.38. Found: C, 92.08; H, 1.80; N, $1.35 \%$.

\section{Acknowledgements}

The authors wish to thank the University of Salento, University of Urbino. "Carlo Bo" and the "Ministero dell'Istruzione, Università e Ricerca" (MIUR) for financial support of the project N. 8206. L. 297, D.M. 593.

\section{References}

1. (a) Attanasi, O. A.; Buratti, S.; Filippone, P. Chim. Ind. 2003, 85. (b) Attanasi, O. A.; Buratti, S.; Filippone, P. Chim. Ind. 1996, 78, 693. (c) Tyman, J. H. P. Synthetic and Natural Phenols, Elsevier:Amsterdam, 1966 and references cited therein. 
2. Hirsch, A.; Berettreich Fullerene-Chemistry; Wiley-VCH: Weinheim, 2005.

3. Hebard, A. F.; Rosseinsky, M. J.; Haddon, R. C.; Murphy, D. W.; Glarum, S. H.; Palstra, T. T. M.; Ramirez, A. P.; Kortan, A. R. Nature 1991, 350, 600.

4. Wang, P.; Metzger, R. M.; Bandow, S.; Maruyama, Y. J. Phys. Chem. 1993, 97, 2926.

5. Wang, P.; Maruyama, Y.; Metzger, R. M. Langmuir 1996, 12, 3932.

6. Thomas, K. G.; Biju, V.; Guldi, D. M.; Kamat, P. V.; George, M. V. J. Phys. Chem. A 1999, 103, 10755.

7. Da-Ros, T.; Prato, M.; Guldi, D. M.; Alessio, E.; Ruzzi, M.; Pasimeni, L. Chem. Commun. 1999, 635.

8. D'Souza, F.; Deviprasad, G. R.; El-Khouly, M. E.; Fujitsuka, M.; Ito, O. J. Am. Chem. Soc. 2001, 123, 5277.

9. Wang, P.; Metzger, R. M.; Chen, B. Thin Solids Films 1998, 327-329, 96.

10. Shi, X.; Caldwell, W. B.; Chen, K.; Mirkin, C. A. J. Am. Chem. Soc. 1994, 116, 11598.

11. Metha, G.; Shing, V. Chem. Soc. Rev. 2002, 31, 324.

12. De la Torre, M. D. L.; Marcorin, G. L.; Pirri, G.; Tomé, A. C.; Silva, A. M. S. ; Cavaleiro, J. A. S. Tetrahedron Lett. 2002, 43, 1689.

13. De la Torre, M. D. L.; Tomé, A. C.; Silva, A. M. S.; Cavaleiro, J. A. S. Tetrahedron Lett. 2002, 43, 4617.

14. Enes, R. F.; Tomé, A. C.; Cavaleiro, J. A. S. Tetrahedron 2005, 61, 1423.

15. Vasapollo, G.; Mele, G.; Longo, L.; Ianne, R.; Gowenlock, B.G.; Orrell, K. G. Tetrahedron Lett. 2002, 43, 4969.

16. Attanasi, O. A.; Del Sole, R.; Filippone, P.; Ianne, R.; Mazzetto, S. E.; Mele, G.; Vasapollo, G. Synlett 2004, 5, 799 .

17. (a) Attanasi, O. A.; Buratti, S.; Filippone, P. Org. Prep. Proced. Int. 1995, 27, 645. (b) De Avellar, I. G. J.; Godoy, K.; de Magalhaes, G. C. J. Braz. Chem. Soc. 2000, 11, 22. (c) Amorati, R.; Attanasi, O. A.; El Ali, B.; Filippone, P.; Mele, G.; Spadavecchia, J.; Vasapollo, G. Synthesis 2002, 2749-2755. (d) Attanasi, O. A.; Beretta, S.; Fiani, C.; Filippone, P.; Mele, G.; Saladino, R. Tetrahedron 2006, 62, 6113. (e) Mele, G.; Vasapollo, G. Mini Rev. Org. Chem. 2008, 5, 243.

18. Attanasi, O. A.; Filippone, P.; Calducci, S. Gazz. Chim. Ital. 1991, 121, 487.

19. (a) Guo, Y. C.; Mele, G.; Martina, F.; Margapoti, E.; Vasapollo, G.; Xiao, W. J. J. Organomet. Chem. 2006, 691, 5383-5390. (b) Mele, G; Li, J., Vasapollo, G. Chimica OggiChemistry Today 2008, 26, 72.

20. (a) Maggini, M.; Scorrano, G.; Prato, M. J. Am. Chem. Soc. 1993, 115, 9798. (b) Prato, M.; Maggini, M. Acc. Chem. Res. 1998, 31, 519.

21. Attanasi, O. A.; Ciccarella, G.; Filippone, P.; Mele, G.; Spadavecchia, J.; Vasapollo, G. J. Porphyrins Phthalocyanines 2003, 7, 52.

22. Attanasi, O. A.; Del Sole, R.; Filippone, P.; Mazzetto, S. E.; Mele, G.; Vasapollo, G. J. Porphyrins Phthalocyanines 2004, 8, 1276. 